\title{
Interactions between platelets and leukocytes in pathogenesis of multiple sclerosis
}

\author{
Angela Dziedzic ${ }^{A-E}$, Michał Bijak ${ }^{A-F}$ \\ Department of General Biochemistry, Faculty of Biology and Environmental Protection, University of Lodz, Poland \\ A - research concept and design; $\mathrm{B}$ - collection and/or assembly of data; $\mathrm{C}$ - data analysis and interpretation; \\ $D$ - writing the article; $E$ - critical revision of the article; $F$ - final approval of the article
}

\section{Address for correspondence \\ Angela Dziedzic \\ E-mail: angela.dziedzic@unilodz.eu}

\section{Funding sources}

This work was supported with funding from scientific research grant from the Polish National Science Centre (No. UMO-2016/21/B/NZ4/00543) as well as with University of Lodz grant (No. 506/1136).

\section{Conflict of interest \\ None declared}

Received on November 20, 2017

Reviewed on January 17, 2018

Accepted on January 18, 2018

Published online on September 6, 2018

\begin{abstract}
Neurodegenerative diseases are an increasing problem in the modern world. Multiple sclerosis (MS) is a major human demyelinating and degenerative disease of the central nervous system (CNS). There are many reports that point to the significant role of platelet-leukocyte interaction in neurodegenerative diseases and cardiovascular disturbances. Epidemiological studies confirm the high risk of cardiovascular diseases in patients with MS. The pathophysiology mechanisms of this multi-component disease are very complex and involve various types of cells. There is increasing evidence that some co-stimulatory pathways affect the function of inflammatory cells, both in the periphery and in the CNS. Interactions of leukocytes and endothelial cells (ECs) could be significantly modulated in the presence of activated blood platelets. The supposed role of activated platelets in the development of vessel inflammatory response is due to their ability to adhere to inflamed EC s or proteins included in the subendothelial layer of the blood vessel wall, as well as to the ability of platelets to form aggregates with leukocytes. Blood platelets are able to directly activate leukocytes through a receptor-dependent mechanism or, indirectly, by biologically active compounds secreted from their granules. Cell-cell interactions provide critical mechanisms by which platelets link thrombosis, inflammation and related processes, such as diapedesis and leukocyte infiltration, to the affected vessel. Determining the relationship between platelet-leukocyte interactions and the development of neuroinflammation in the course of MS may provide new therapeutic targets in the future.
\end{abstract}

Key words: blood platelets, multiple sclerosis, neuroinflammation, platelet-leukocyte aggregates

Cite as

Dziedzic A, Bijak M. Interactions between platelets and leukocytes in pathogenesis of multiple sclerosis. Adv Clin Exp Med. 2019;28(2):277-285. doi:10.17219/acem/83588

DOI

10.17219/acem/83588

\section{Copyright}

Copyright by Author(s)

This is an article distributed under the terms of the

Creative Commons Attribution Non-Commercial License

(http://creativecommons.org/licenses/by-nc-nd/4.0/) 


\section{Introduction}

Neuroinflammation is an inflammation of the nervous tissue, whereas neurodegeneration is the progressive loss of structure and function of neurons in specific regions of the central nervous system (CNS), which is a significant microenvironment for the proper functioning of neurons. ${ }^{1,2}$ The neurodegeneration is a term that embraces a broad range of clinical diseases with chronic inflammation, of which the most common are multiple sclerosis (MS), Alzheimer's disease (AD) and Parkinson's disease (PD). Multiple sclerosis is one of the most frequently occurring disorders of neurological disability among young adults, typically appearing between the age of 20 and 40. It affects around 2.3 million people worldwide, of whom roughly 600,000 live in Europe. ${ }^{3}$ Multiple sclerosis is a chronic, demyelinating neurodegenerative disease of the CNS with a complex of different clinical courses and a number of pathophysiological mechanisms, such as axonal/neuronal damage, neuroinflammation, demyelination, gliosis, and remyelination. The pathomechanism of MS is based on alterations of the immune system together with biochemical disturbances and a disruption of blood-brain barrier (BBB). The blood-brain barrier is a structural complex composed of matrix components and several cell types (e.g., endothelial cells (ECs), pericytes, perivascular microglia, and astrocytes). These barriers are responsible for supporting metabolic homeostasis and immune regulation of $\mathrm{CNS}{ }^{4}$

Blood platelets are a key element in maintaining physiological hemostasis. Hemostasis is a complex of physiological processes that ensure vascular tightness and circulating blood flow, and inhibit bleeding after breaking the continuity of a blood vessel. Numerous interacting elements are involved in the maintenance of normal hemostasis, including blood vessel wall, blood platelets, coagulation cascade, fibrinolytic system, and phagocytic cells. Damage to ECs and exposure of the thrombogenic adhesion proteins lead to platelet activation and initiation of coagulation cascade. This is accomplished by forming a stable platelet plug via adhesion to the revealed subendothelial matrix proteins (e.g., von Willebrand factor (vWF) and collagen). The platelet membrane is rich in glycoproteins (GP Ia-IIa $/ \alpha_{2} \beta_{1}$, GP Ib-V-IX, GP Ic-IIa $/ \alpha_{5} \beta_{1}$, GP IIb-IIIa $/ \alpha_{\text {IIb }} \beta_{3}$, and GP IV), which bind to activate platelets and serve a primarily adhesive function. Throughout the interaction with the ECs, platelets become partly active, accordingly changing their shape from a discoid to a pseudopodia state. ${ }^{5}$ Activation results in platelet aggregation by binding GP IIb-IIIa to fibrinogen, which ultimately results in the formation of a stable platelet plug. ${ }^{6}$

Interestingly, epidemiological studies confirm that there is an increased risk of cardiovascular disease in patients with MS, especially of prothrombotic events fairly associated with decreased hemostatic platelet activity. Platelets have been recognized as the smallest blood cells that fulfill a complex role in hemostasis/thrombosis and also in inflammation. Platelets possess a large variety of molecules stored in their granules, including numerous membrane receptors, immunomodulatory mediators and cell adhesion molecules. There is a link between platelets and pathophysiology of MS, and these small cells may be a key player in neuroinflammation. Activated platelets are responsible for the interaction with leukocytes and initiate increased infiltration of autoreactive $\mathrm{T}$ cells, which form new neuroinflammatory lesions in CNS.?

\section{Biological active substances derived from blood platelets}

Platelets are one of the most abundant cells in the circulatory system, which contain numerous biologically active compounds that are stored in their granules and release them during activation. Those signaling substances play a key role in the continuous communication of platelets with other platelets, immune cells and ECs. The capacity of blood platelets to secrete from their storage vast amounts of cytokines, chemokines and other related molecules appears closely related to their role in hemostasis and inflammation. Circulating platelets are inactive until they adhere to exposed subendothelial matrix proteins or are stimulated by soluble agonists. Each activating event is associated with a change in platelet shape, cytoskeletal reorganization and the secretion of platelet granules. ${ }^{8}$ Proteins involved in the behavior of physiological hemostasis include fibrinogen, vWF, vitronectin, fibronectin, thrombospondin, factor V, factor VIII, coagulation factors, fibrinolytic inhibitors, mitogens, adhesion molecules, and membrane glycoproteins (GP Ib, IIb and IIIa). ${ }^{9}$ During activation, the platelets expose a vast number of surface-bound molecules stored in their $\alpha$-granules, such as inflammatory cytokines (e.g., CD40L or interleukin (IL)-1ß), resulting in endothelial activation and the secretion of chemoattractants, such as platelet activation factor (PAF), chemokines platelet factor (PF4) and RANTES/CCL5 (regulated on activation normal T-cells expressed and secreted). Furthermore, dense granule constituents, such as serotonin, adenosine 5'-triphosphate (ATP), adenosine 5'-diphosphate (ADP), polyphosphates, and glutamate, are known as modifiers of platelet activation and thrombus formation, but many have also immune cell-modifying effects. ${ }^{10}$ PF4/CXCL4 is one of the most abundant proteins belonging to the $\mathrm{CXC}$ subfamily of chemokines, synthesized in megakaryocytes, enclosed in vesicles and transferred to the $\alpha$-granules from which it is secreted during platelet activation (it represents $25 \%$ of $\alpha$-granule storage). Basal concentration of PF4 in human plasma is $2-20 \mathrm{ng} / \mathrm{mL}$. The PF4 has many cell targets, such as neutrophils, monocytes/macrophages and T-cells. It has a wide scope of activities related to innate immunity, including effects on monocyte and neutrophil chemotaxis. ${ }^{11}$ The PF4 initiates a signal transduction cascade in monocytes, leading to the induction of a wide spectrum of functions, including phagocytosis, respiratory burst, monocyte 
survival, and the secretion of cytokines. It activates neutrophils by binding to the proteoglycan chondroitin sulfate on the surface of neutrophils. The PF4 can promote the release of neutrophil granules and enhanced adhesion neutrophils to ECs. It induces monocyte release of cytokines, such as CXCL3, CXCL8, IL- $1 \alpha$, IL-1 $\beta$, IL-6, IL-19, tumor necrosis factor $\alpha$ (TNF- $\alpha$ ), CCL2, CCL3, and CCL22. ${ }^{12}$ Moreover, CXCL4 also expresses immunomodulatory activities, such as downregulation of interferon production by type 1 T-helper (Th1) cells, up-regulation of IL-4, IL-5, and IL-13 in type 2 T-helper (Th2) cells. The PF4 inhibits the production of Th1 cytokines and promotes the production of Th2 cytokines through the interaction with CXCR3-B receptor. ${ }^{13}$ Chemokine (C-C motif) ligand 5 (CCL5), also known as RANTES (regulated on activation, normal $\mathrm{T}$ cell expressed and secreted), is a CC-subfamily of chemokines produced by extravascular cells. Chemotaxis induced by RANTES is dependent on the activation of p38 mitogen-activated protein kinase (MAPK) pathway. The RANTES and monocyte chemoattractant protein-1 (MCP-1/CCL2) induce tissue fibroblasts to produce IL-6 and IL-8. The RANTES can also promote IL-2 and IL-5 expression in T cells. The synthesis of IL- 1 , TNF- $\alpha$, and IL- 6 can be stimulated by macrophages-derived inflammatory protein-1 (MIP-1/CCL3). ${ }^{14}$ Schober et al. observed that the blockade of RANTES is a powerful tool to inhibit monocyte recruitment on different ECs types, which impairs macrophage infiltration. ${ }^{15}$

Furthermore, platelet-derived IL-1 $\alpha$ (but not IL-1 $\beta$ ) stored in $\alpha$-granules is capable of activating peripheral ECs, and mediates platelets-promoted leukocyte infiltration in peripheral tissues, which also enhances transendothelial migration of neutrophils (TEM). Measurements of platelet and leukocyte IL-1 $\alpha$ level indicate that the expression of platelet-derived IL-1 $\alpha$ was 3.6-fold higher compared with the levels expressed by leukocyte populations, suggesting that blood platelets are the main source of IL- $1 \alpha$. The study conducted by Thornton et al. showed that platelet-secreted IL- $1 \alpha$ drives endothelial activation in a mouse brain, which can lead to the release of endothelial intercellular adhesion molecule 1 (ICAM-1), vascular cell adhesion protein 1 (VCAM-1) and CXCL1 chemokine. Low concentrations of IL- $1 \alpha$ released from platelets during activation induce significant brain endothelium activation. Mouse brain endothelium is very sensitive to IL- $1 \alpha$, with concentrations as low as $1.5 \mathrm{pM}$ being able to induce CXCL1 secretion and enhance CAM expression, which suggests that local release of platelet IL-1 $\alpha$ could mediate cerebrovascular inflammation in vivo. ${ }^{16}$

\section{Interactions between platelets and leukocytes in inflammation}

The proinflammatory activity of blood platelets occurs through a variety of mechanisms, including receptor-ligand connection and activation of different cells, as well as through the release of severe biologically active molecules stored in their granules. This bidirectional process is called cellular cross-talk. ${ }^{17}$ Platelets possess a wide array of glycoprotein membrane receptors that are crucial for their interactions with other platelets, leukocytes and ECs. Platelets are expressed by a few members of the $\beta 1$-subfamily integrins $\left(\alpha_{\text {II }} \beta_{1}\right.$ and $\left.\alpha_{v} \beta_{1}\right)$, and $\beta 3$-subfamily integrins $\left(\alpha_{\mathrm{v}} \beta_{3}\right.$ and $\left.\alpha_{\mathrm{IIb}} \beta_{3}\right)$, that support platelet adhesion to the endothelial cell-matrix proteins, such as collagen, fibronectin and laminin. ${ }^{18} \mathrm{GP}$ IIb-IIIa (also known as $\alpha_{\text {IIb }} \beta_{3}$ ) is a receptor for fibrinogen, vWF, fibronectin, and vitronectin, which is definitely a major initiator of platelet aggregation. The formation of hemostatic plug is determined, to a large extent, by the integrin GP IIb-IIIa-mediated interactions of platelets with their receptors. ${ }^{19}$ There are several possible connections between platelets and leukocytes. The main interaction between platelets and leukocytes, which is responsible for the formation of platelet-leukocyte aggregates (PLAs), is the connection between platelet P-selectin (CD62P) and leukocyte P-selectin glycoprotein ligand-1 (PSGL-1). Platelets are a major source of $\mathrm{CD} 62 \mathrm{P}$ in the circulatory system. ${ }^{20}$ $\mathrm{CD} 62 \mathrm{P}$ is a membrane glycoprotein heavily present in platelet $\alpha$-granules and Weibel-Palade bodies in ECs that is exposed on the platelet surface during activation. CD62P/ PSGL-1 interaction has a functionally key role in leukocyte rolling and adhesion to platelets and endothelium, which are the main step in the process of leukocyte recruitment and extravasation. ${ }^{21}$ PSGL-1 protein is also expressed on all T-cells types. In murine models, CD62P-positive platelets mediate adhesion of leukocytes into endothelium leading to the recruitment of T-cells to the place of inflammation/ injury. ${ }^{22}$ Individual leukocyte subpopulation demonstrates different affinity for CD62P present on platelets surface. From all leukocytes, monocytes show the greatest affinity for platelet CD62P, and thus to the formation of plateletleukocyte aggregates. ${ }^{23}$ It is known that PSGL-1 is likely to be a prevailing ligand for CD62P. Interestingly, Hirata et al. have confirmed that PSGL-1 on Th1 cells functions is the only ligand for CD62P and E-selectin in vivo. ${ }^{24}$ It is worth noticing that most lymphocytes express PSGL-1 on their surface, but only $10-20 \%$ of them are actually able to bind platelet CD62P. To make a connection with P-selectin, PSGL-1 ligand needs to be post-translationally modified by protein glycosylation and tyrosine sulfation. ${ }^{25}$ Abundant adhesion connections have been identified and implicated in the leukocyte transmigration process. Leukocyte recruitment occurs at the sites of vascular injury, where the subendothelial proteins have been externalized and activated platelets have been deposited. The initial tethering and rolling of leukocytes are followed by their strong adhesion and platelet migration, which depends on macrophage antigen 1 (Mac-1, $\left.\alpha_{M} \beta_{2}, C D 11 b / C D 18\right)$. The initial link between platelets and leukocytes (CD62P/PSGL-1) leads to increased expression of Mac-1 on leukocytes, which itself supports interactions with platelets. Mac-1 can also interact with GP IbA present on platelets, which mediate adhesive 
interactions between leukocytes and platelets. Wang et al. had observed that leukocyte recruitment markedly had been decreased by $73 \%$ in the case of CD62P deficiency on the platelet surface and $67 \%$ in the lack of Mac-1, suggesting that leukocyte recruitment is largely dependent on the aforementioned interactions. ${ }^{26}$ Simon et al. observed that the binding of neutrophils from wild-type $\left(\mathrm{Mac}^{-1^{+/}}\right)$ and Mac-1-deficient (Mac-1 ${ }^{-/-}$) mice to GP IbA. Mac-1 ${ }^{+/+}$ neutrophils bound to adherent platelet; however, $\mathrm{Mac}^{-1^{-/}}$ neutrophils demonstrated markedly reduced adhesion to platelets, which implies that the predominant interaction between neutrophils and platelets occurs between Mac-1 and GP IbA. The specificity of the interaction was that neutrophils from wild-type mice (but not from $\mathrm{Mac}-1^{-/-}$mice) bound to adherent platelet GP IbA. These studies demonstrate that GPIbA is a physiologically relevant ligand for Mac-1 and is critical to leukocyte function in vivo. ${ }^{27}$ Binding of platelet endothelial cell adhesion molecule-1 (PECAM-1/CD31), ICAM-2/CD102, CD99, and members of the junctional adhesion molecule (JAM) family (e.g., JAM-A and JAM-C) with leukocyte ligands may assist in leukocyte transendothelial migration. ICAM-2, JAM-A and PECAM-1 are all members of the immunoglobulin superfamily of adhesion molecules. Woodfin et al. have observed that the lack of ICAM-2 in a murine asthma model has delayed the increase in eosinophil infiltration. Those findings indicate that the adhesion molecules ICAM-2, JAM-A and PECAM-1 can support neutrophil emigration through venular walls in a highly organized and regulated manner after local ECs activation. These studies may suggest that adhesion molecules may play a crucial role in mediating physiologic leukocyte infiltration as part of the immune response. There is a vast number of evidence demonstrating that the EC molecules support neutrophil transmigration in vivo in a stimulus-dependent way. ${ }^{28}$

Despite the lack of cell nucleus, the platelets contain stable mRNA transcripts that can synthesize de novo the proteins involved in maintaining physiological hemostasis and inflammatory process. For hemostasis, platelets synthesized proteins, including coagulation factor XI, tissue factor (TF), GP Iib-IIIa, ${ }^{29}$ and cyclooxygenase (COX). ${ }^{29,30}$ Proteins involved in inflammation, synthesized by platelets, are IL- $1 \beta$ and chemokine RANTES. ${ }^{31}$ The mRNA transcript for IL- $1 \beta$ are constitutively present in platelet polysomes. Platelet activation induces a rapid and sustained synthesis of pro-IL-1 $\beta$ protein. A portion of the IL-1 $\beta$ is shed in its mature form in membrane microvesicles and induces adhesiveness of human ECs for neutrophils. Lindeman et al. recently found that IL-1 $\beta$ acts in concert with CD62P to induce expression of COX-2, when human monocytes interact with activated platelets. ${ }^{32}$ Interleukin 1- $\beta$ and IL1R1 are associated with the innate immune system and can regulate both megakaryocyte and platelet functions in human models. Platelet IL-1 $\beta$ through IL1R1 can stimulate megakaryocyte maturation and modify the RNA profiles in the evolving platelets to be prothrombotic and proinflammatory. The presence of IL-1 $\beta$ stimulates ECs to secrete their chemotactic proteins. Interleukin $1 \beta$ affects the endothelium layer and smooth muscle cells by stimulating inflammation, and initiates platelet adhesion to ECs. NFKB pathway can be activated by myeloid differentiation primary response gene 88 (MYD88), which stimulates it through IL1R1 and its ligand IL-1 $\beta$. This process also enhances the expression of other immune-related cytokines, growth factors and adhesion molecules. ${ }^{33}$ Studies conducted by Dixon et al. show that activated platelet interactions with PSGL-1 on the monocytes enhance COX-2 expression and increase synthesis of prostaglandin E2 by monocytes via molecular mechanisms involving cellular adhesion and cytokine signaling. ${ }^{30}$

\section{Role of blood platelets in neuro-inflammation and neurodegenerative diseases}

One of the major hallmarks of MS is chronic inflammation. There are a vast number of different inflammatory effectors, which can be responsible for the neuronal/ axonal pathology in MS. ${ }^{34}$ Multiple sclerosis is a chronic inflammatory disease of the CNS. The association between chronic inflammation and subsequent cancer is well-established, and chronic inflammation is thought to have a role in both the initiation and promotion of neoplasms. Chronic inflammation has been hypothesized to play a role in triggering clonal evolution in myeloproliferative neoplasms (MPNs) which are stem-cell-derived disorders causing overproduction of 1 or more of blood elements, including essential thrombocythemia. In this context, it is important to note the unusual concurrence of MS and MPNs in 5 patients from a localized geographic area in Denmark. It is highly interesting to recognize the potentially common factors predisposing patients to both MS and MPNs. ${ }^{35}$ Further studies are needed to clarify if any association between MS and MPNs indeed exists, especially that essential thrombocythemia may contribute to the increased risk of cardiovascular disease, especially ischemic stroke, myocardial infarction and thrombosis observed in MS. ${ }^{36-38}$ Symptoms of MPNs associated with essential thrombocythemia may be underdiagnosed in MS patients, since many different possible reasons of high risk of tromboembolism events have inclinations in MS. Multiple sclerosis patients lead a more sedentary lifestyle, which promotes the occurrence of stroke, ${ }^{39}$ and, above all, is associated with the risk of venous thrombosis. ${ }^{40,41}$ Treatments for MS may also increase the risk for cardiovascular diseases. ${ }^{42}$

There are a number of studies which indicate that platelets, apart from their major role in cellular hemostasis, also participate in the development of autoimmune 
mechanisms, neurodegeneration and neuroinflammation. ${ }^{43,44}$ There are many reports suggesting that platelets are chronically activated in neurodegenerative diseases. Platelet activation, degranulation and platelet-leukocyte interactions may affect the pathophysiology of neurodegenerative diseases, including MS.

There is quite a long history of studies conducted on the pathogenesis of MS, dating back to the middle of the previous century. Nathanson and Savitsky were the first to indicate increased platelet adhesiveness in patients suffering from MS. ${ }^{45}$ In the following years, platelet overactivation in MS was confirmed. Sheremata et al., using a flow cytometer, showed increased platelet activation in MS, expressed in a high percentage of microparticles and platelet aggregates, and increased surface exposure of P-selectin, a platelet activation marker that plays an important role in their interaction with ECs and leukocytes. ${ }^{46}$ The latest research also confirms that MS patients displayed higher levels of platelet activation parameters in the circulation. ${ }^{47,48}$ Moreover, cyclooxygenase-dependent arachidonic acid metabolism (a key pathway of platelet activation) is significantly increased in blood platelets of patients with MS. ${ }^{49}$

The chronic activation of platelets in MS has been proven, although their role in this pathology still needs to be clarified. Patients with MS experience various generalized disruptions, depending on the number and placement of active lesions in the CNS. In physiological conditions, there is an extremely low level of immune cells, such as neutrophils and lymphocytes, in CNS. Platelets may contribute to inflammation by enhancing leukocytes adhesion to ECs and promote CNS inflammation. Moreover, Langer et al. proved that platelets are trapped in chronic active demyelinating MS lesion. They also observed that after inhibiting the main platelets receptors - GP IIb/IIIa (responsible for binding of Fg and aggregation), the paralysis and experimental autoimmune encephalomyelitis (EAE) were respectively ameliorated and reduced. Similarly, reduction of platelet count markedly alleviated the symptoms and inhibited the inflammation process. ${ }^{50,51}$ The hematological profile of patients with MS indicates an increased number of platelets in the circulation of patients, which may suggest their immunological involvement. ${ }^{52}$ Therefore, the decrease in platelet count in MS patients may be a positive force for protecting against the development of the disease. Farrokhi et al. showed a statistically significant decrease in platelet level for patients taking fingolimod oral medication (reducing classic autoimmune cells). Platelet count was decreased in the entire cohort taking fingolimod for 1 month. ${ }^{53}$ Probably, a similar thrombocytopenia effect may be demonstrated by the treatment with alemtuzumab. ${ }^{54}$

The direct interaction of platelets with ECs and inflammatory cells promotes leukocyte recruitment to the inflamed tissue through platelet receptors. The pathomechanism of MS involved alterations of the immune cells together with biochemical disturbances and a disruption of the BBB. ${ }^{1}$ Enormous influx of inflammatory cells (lymphocytes, neutrophils, monocytes, and macrophages) recruitment, along with the proinflammatory stimulation of microglia cells, altogether result in the destruction of the myelin sheath, which accelerates demyelinating lesions. ${ }^{50}$

CD40 ligand (CD40L; CD154) and its receptor CD40, co-stimulatory molecules of the TNF-family receptor, have an important role in inflammation. CD40L is expressed in many cell types, such as platelets, T-cells, macrophages, neutrophils, smooth muscle cells, and ECs. CD40L affect platelet-platelet, platelet-leukocyte and leukocyte-endothelium interactions. CD40L is responsible for transferring adhesion signals into the cell "outside-in" signaling by reaction within ECs, and integrins that mediate platelet-endothelium adhesion. Platelet CD40L enhances the expression of endothelial adhesion receptors, including E-selectin, VCAM-1, ICAM-1, as well as chemokines (e.g. CCL2, CXCL4 and CCL5) and MMPs (MMP-1, MMP-2, MMP-9, and MMP-14). ${ }^{55}$ All these molecules can mediate the attachment of neutrophils, monocytes and lymphocytes to the inflamed vessel wall. Ligation of CD40 on ECs by CD40L, expressed on the surface of activated platelets, increases the release of IL-8 and MCP-1, which is the principal chemo-attractants for neutrophils and monocytes recruitment. ${ }^{56}$ The CD40/CD40L pathway at sites of immune reactivity in chronic inflammatory and autoimmune disorders is constantly activated, as shown by the presence of abundant CD40-positive and CD40L-positive cells. This pathway is designed to generate signals for the recruitment of leukocytes at the site of inflammation. CD40L molecules are highly expressed in activated T-cells and active platelets, and they induce ECs to secrete chemokines and to express adhesion molecules. CD40 is present in B cells, monocytes, macrophages and ECs. Firstly, platelets are activated through binding with CD40L-positive T-cells. Secondly, through the release of RANTES, platelets can recruit more T-cells to create an amplification loop promoting leukocytes' recruitment to the site of inflammation. CD40L-positive T-cells induced platelet activation resulting in RANTES release, which binds to ECs and mediates T-cell recruitment. Furthermore, CD62P/PSGL-1 connection could increase the intensification and maintenance of immune and inflammatory responses. ${ }^{17}$ Lievens et al. have demonstrated that platelet CD40L promotes platelet and leukocyte adhesion to the endothelium and stimulates the formation of PLAs. Activated platelets can indirectly support leukocyte recruitment via formation of PLAs. The lowest numbers of PLAs were detected with both platelets and leukocytes lacking CD40L, which indicates that the interaction between platelet CD40L and leukocyte CD40 facilitates PLAs formation. Repeated intravenous injection of activated CD40L-negative platelets into ApoEnegative mice prevented the increase of atherosclerosis and the disruption of T-cell homeostasis that was observed after injection of activated CD40L-positive platelets. 
This study suggests that CD40L plays an important role in hemostasis/thrombosis and in modulating immune responses. ${ }^{57}$

Active tissue injury in MS lesions is associated with activated microglia and massive infiltration of macrophages. Th cells are a type of T-cell that plays a major role in the immune system, especially in the adaptive immune system. These cells secrete a number cytokines, such as interferon $\gamma$ (IFN- $\gamma$ ) and IL-2, which activate other immune cells and enhance vascular inflammation. Th cells are well-known to potentiate atherogenesis, while regulatory T-cells (Tregs) display atheroprotective effects. Platelets can form PLAs, which may facilitate lymphocyte into arterial thrombi, and gatheringon ECs. Platelets enhance Thcell cytokine production through CD40-CD40L binding. Platelets may promote CD4-positive T-cell differentiation by inhibiting their proliferation. Platelets enhance the production of Th1 and promote both Tregs and Th17 differentiation, as evidenced by elevated level of IL-10 and IL-17 production. Th1 and Th17 are 2 basically counteracting pathways of CD4-positive T-cell differentiation. ${ }^{58,59}$ In most EAE models, which are driven by Th1 or Th17 cells, inflammation starts with profound infiltration of the tissue by the major histocompatibility complex (MHC) Class II restricted CD4-positive T-cells, which is followed by microglia activation and macrophage recruitment into the lesions. ${ }^{43}$ In EAE and MS, Th1 and Th17 cells infiltrate into the CNS through the BBB, where they become reactivated and initiate the destruction of myelin sheath (demyelination) and axonal/neuronal degeneration in MS. ${ }^{2,60}$ It has also been demonstrated that IL-1 $\beta$, IL-6, IL-23, and the transforming growth factor (TGF- $\beta$ ) are crucial for human Th17 differentiation from naive CD4-positive and CD45-positive peripheral blood lymphocytes. The TGF- $\beta$ and IL-21 promote the polarization of Th17 cells from human naive CD4-positive T-cells. ${ }^{61}$ Th1 cells also secrete proinflammatory cytokines, such as IL-2 and TNF- $\alpha$, which can activate other lesional cells. Th17 cell secrets cytokine, including IL-17, TNF- $\alpha$ and IL-6, which have both overlapping and distinct roles in neuroinflammation. ${ }^{62}$ Interleukin 17 enhances the production of proinflammatory cytokines and chemokines by other cells via activating the nuclear factor kappa B (NFkB) pathway. Interleukin 17 appears to play an essential role in the pathogenesis of chronic inflammatory disorders and in many autoimmune diseases, including MS. ${ }^{63}$ T-effector-cell responses and cytokine production is modulated by platelet-derived soluble mediators, such as IL-1 $\beta$, PAF and PF4, which can stimulate the differentiation of T-cells into pathogenic Th1, Th17 and IFN- $\gamma$ / IL-17-producing CD4-positive T cells. At the later stages of these neurodegenerative diseases, platelets became exhausted in their capacity to produce proinflammatory factors and to stimulate CD4-positive T-cells, but strongly increase their ability to form complexes with CD4-positive T-cells. Formation of platelet-CD4-positive T-cell complexes involve the interaction of CD62P in activated platelets with the adhesion molecule CD166 in activated CD4-positive T-cells, contributing to the downmodulation of CD4-positive T-cell activation, the proliferation and production of IFN- $\gamma^{47}$

Patients with MS displayed increased numbers of proinflammatory cytokine producing Tregs. Furthermore, Forkhead box P3 (FOXP3) Tregs are fundamental for immunity and homeostasis. FOXP3 expression is essential for Treg suppressor function and Treg stability. The human peripheral blood CD4-positive T-cells comprise 5\% of expression of naturally occurring FOXP3 in Tregs. ${ }^{64}$

Activated platelets release plasma membrane plateletderived microparticles (PMPs) into the circulatory system. Platelet-derived microparticles play an important role in the regulation of immunity by transferring membrane receptors and microRNA to other circulatory cells. These platelets and megakaryocyte-derived microparticles are identified by the expression of platelet activation markers, such as CD62P and/or CD63. Thus, cell proliferation may be one of the mechanisms, whereby PMPs prevent IL-17 production by Tregs. ${ }^{64}$

During circulation, platelets become reactive and release different types of constituents stored in their granules. An increased level of platelet-derived active compounds (PF4, CD40L, PAF, MMPs, serotonin, RANTES, and $5 \mathrm{HT}$ ) has been taken as a marker of platelet activation in MS. ${ }^{56,65}$ Duerschmied et al. hypothesized that peripheral serotonin/5-hydroxytryptamine (5HT) may be involved in the migration of leukocytes during acute inflammatory processes. They report that the targeted recruitment of neutrophils into inflammation site is promoted probably by platelet-derived $5 \mathrm{HT}$. Thus, they play an important role in neutrophil homeostasis that is reminiscent of the phenotype of CD62P-deficient mice. ${ }^{66}$ Blood platelets are the exclusive source of $5 \mathrm{HT}$, which is a main neurotransmitter that can directly affect the functions of neurons in the CNS and can modulate the functions of the immune cells. It has an important role in neutrophil rolling and adhesion to the endothelium; therefore, it can recruit neutrophils at the site of inflammation. It can enhance the stimulation of CD4-positive T-cells by a monocyte. ${ }^{21}$ Adenosine 5'-triphosphate can modulate inflammatory pathways by activating dendritic cells. The ATP signaling through T cell P2X7 receptor can increase the differentiation of CD4-positive Th cells toward a proinflammatory Th17 cell type. Polyphosphates may induce NFkB pathway activation and the expression of endothelial adhesion molecules. ${ }^{67}$ The glutamate transmission in the striatum and dendritic spine loss lead to the chronic inflammation in experimental autoimmune encephalomyelitis (EAE) and murine model of MS. It is strongly independent of demyelination and associated with a huge release of TNF- $\alpha$ from activated microglia cells. Excitotoxicity mediated from glutamate is emerging as a critical determinant of axonal/neuronal injury in MS, and T-cell migration. The level 
of glutamate have been found to be meaningfully higher in the brains of MS patients. ${ }^{68}$

Matrix metalloproteinases (MMPs) are enzymes that play a major role in basic cell behaviors, such as cell proliferation, migration, differentiation, angiogenesis, apoptosis, and host defense. There are 3 different mechanisms of directing MMPs activity to specific target sites. Firstly, platelets can concentrate the proteolytic activity of inflammatory cells at the site of a vascular injury. Secondly, by forming cellular aggregates with leukocytes, platelets may stimulate the production of MMPs in these cells. Lastly, a great deal of evidence indicates direct platelet expression of couple MMPs, including MMP-1, MMP-2, MMP-3, and MMP-14. ${ }^{69}$ All protein components of extracellular matrix (ECM) can be degraded by MMPs. Another negative function of MMPs in MS is thought to include the disruption of the BBB. In vitro, inhibitors of MMPs block the transmigration of T-cells across basement membrane matrices. The abundance of MMPs expression occurs in monocytes, which emphasizes the key role of monocytes, crucial for evolving the neuroinflammatory process in MS. Bar-Or et al. have reported an increased level of MMP-2, MMP-7, MMP-9, and MMP-12 in MS brain tissue. Young mice that are genetically deficient in MMP-9 are relatively resistant to EAE induction compared with wild-type mice. ${ }^{70}$

Experimental studies conducted by Saluk-Juszczak et al. showed that platelets are a source of reactive oxygen species (ROS). Platelet activation by the typical agonists leads to the production and/or release of TXA $\mathrm{TX}_{2}, \mathrm{ADP}$ and ROS, which activates unstimulated platelets. For example, the burst of $\mathrm{H}_{2} \mathrm{O}_{2}$ enhances the formation of platelet aggregates by phospholipid-derived arachidonate pathway. ${ }^{71}$ Reactive oxygen species are natural products of oxidative phosphorylation, but can also be generated by activated inflammatory cells, including macrophages and microglia. Markers of oxidative stress may be different across MS lesions. Many studies suggest that antioxidant enzymes activity is correlated with the presence of oxidative damage and neuroinflammation. ${ }^{72}$

\section{Conclusions}

Chronic neurodegeneration diseases are a serious problem, despite the advanced development of medicine. Multiple sclerosis is one of the most common causes of neurological disability among young adults. Knowledge about the pathophysiology of this disease is insufficient and still needs to be clarified. There is a great deal of evidence to support the significant interaction between platelets and leukocytes in neurodegenerative diseases, including MS. There are many studies which indicate that platelets are highly activated in some patients with MS. Platelets are a rich source of proinflammatory agents and are capable of interacting with leukocytes. The interaction between platelets and leukocytes is based on increased leukocyte recruitment through $\mathrm{BBB}$, and enhanced release of proinflammatory molecules from leukocyte and platelet granules. On the MS example, the major migration of leukocytes to the CNS can cause a temporary loss of integrity by $\mathrm{BBB}$, which contributes to damage and neuronal death. Accurate knowledge and determination of the relationship between platelet interaction in immune cells and the mediators of inflammation in neurodegenerative diseases is a challenge for modern research, which may provide new therapeutic targets in the future. Understanding platelet physiology in the pathobiological processes associated with venules in the inflamed brain will provide new targets for therapy in MS.

\section{References}

1. Waubant E. Biomarkers indicative of blood-brain barrier disruption in multiple sclerosis. Dis Markers. 2006;22(4):235-244.

2. Chen W, Zhang X, Huang W. Role of neuroinflammation in neurodegenerative diseases (review). Mol Med Rep. 2016;13(4):3391-3396.

3. Brola W, Sobolewski P, Flaga S, et al. Prevalence and incidence of multiple sclerosis in central Poland, 2010-2014. BMC Neurol. 2016;16:134.

4. Wachowicz B, Morel A, Miller E, Saluk J. The physiology of blood platelets and changes of their biological activities in multiple sclerosis. Acta Neurobiol Exp (Wars). 2016;76(4):269-281.

5. Dziedzic A, Idzikowska K, Saluk J. Tworzenie się kompleksów płytkowo-leukocytarnych we krwi obwodowej u pacjentów z ostrym zespołem wieńcowym. In: Szklarczyk M, Bajek E, eds. Diagnostyka, profilaktyka, leczenie - najnowsze doniesienia. Vol. II. Lublin, Poland: Wydawnictwo Naukowe Tygiel; 2017:42-50.

6. Drelich DA, Bray PF. The traditional role of platelets in hemostasis (Chapter 2). In: Kerrigan S, Moran N, eds. The Non-Thrombotic Role of Platelets in Health and Disease. London, UK: IntechOpen Ltd.; 2015. doi:10.5772/58357

7. Morel A, Rywaniak J, Bijak M, Miller E, Niwald M, Saluk J. Flow cytometric analysis reveals the high levels of platelet activation parameters in circulation of multiple sclerosis patients. Mol Cell Biochem. 2017;430(1-2):69-80.

8. Elzey BD, Tian J, Jensen RJ, et al. Platelet-mediated modulation of adaptive immunity: A communication link between innate and adaptive immune compartments. Immunity. 2003;19(1):9-19.

9. Bijak M, Saluk J, Ponczek M, Nowak P, Wachowicz B. The synthesis of proteins in unnucleated blood platelets [in Polish]. Postepy Hig Med Dosw. 2013;67:672-679.

10. King SM, McNamee RA, Houng AK, Patel R, Brands M, Reed GL. Platelet dense granule secretion plays a critical role in thrombosis and subsequent vascular remodeling in atherosclerotic mice. Circulation. 2009;120(9):785-791.

11. Srivastava K, Cockburn IA, Swaim AM, et al. Platelet factor 4 mediates inflammation in cerebral malaria. Cell Host Microbe. 2008;4(2):179-187.

12. Kasper B, Brandt E, Brandau S, Petersen F. Platelet factor 4 (CXC chemokine ligand 4) differentially regulates respiratory burst, survival, and cytokine expression of human monocytes by using distinct signaling pathways. J Immunol. 2007;179(4):2584-2591.

13. Lasagni L, Grepin R, Mazzinghi B, et al. PF-4/CXCL4 and CXCL41 exhibit distinct subcellular localization and a differentially regulated mechanism of secretion. Blood. 2007;109(10):4127-4134.

14. Shahrara S, Park CC, Temkin V, Jarvis JW, Volin MV, Pope RM. RANTES modulates TLR-4-induced cytokine secretion in human peripheral blood monocytes. J Immunol. 2006;177(8):5077-5087.

15. Schober A, Manka D, von Hundelshausen $P$, et al. Deposition of platelet RANTES triggering monocyte recruitment requires P-selectin and is involved in neointima formation after arterial injury. Circulation. 2002;106(12):1523-1529.

16. Thornton P, McColl BW, Greenhalgh A, Denes A, Allan SM, Rothwell NJ. Platelet interleukin-1alpha drives cerebrovascular inflammation. Blood. 2010;115(17):3632-3639. 
17. Danese $S$, de la Motte $C$, Rivera Reyes BM, Sans M, Levine AD, Fiocchi $C$. Cutting edge: $T$ cells trigger CD40-dependent platelet activation and granular RANTES release: A novel pathway for immune response amplification. J Immunol. 2004;172(4):2011-2015.

18. Bennett JS. Structure and function of the platelet integrin allb $\beta 3$. J Clin Invest. 2005;115(12):3363-3369.

19. Höök P, Litvinov RI, Kim OV, et al. Strong binding of platelet integrin allb $\beta 3$ to fibrin clots: Potential target to destabilize thrombi. Sci Rep. 2017;7(1):13001. doi:10.1038/s41598-017-12615-w

20. von Hundelshausen $P$, Weber C. Platelets as immune cells: Bridging inflammation and cardiovascular disease. Circ Res. 2007;100(1):27-40.

21. Lam FW, Vijayan KV, Rumbaut RE. Platelets and their interactions with other immune cells. Compr Physiol. 2015;5(3):1265-1280.

22. Green SA, Smith M, Hasley RB, et al. Activated platelet-T-cell conjugates in peripheral blood of patients with HIV infection: Coupling coagulation/inflammation and T cells. AIDS. 2015;29(11):1297-1308.

23. Schrottmaier WC, Kral JB, Badrnya S, Assinger A. Aspirin and P2Y12 inhibitors in platelet-mediated activation of neutrophils and monocytes. Thromb Haemost. 2015;114(3):478-489.

24. Hirata T, Merrill-Skoloff G, Aab M, Yang J, Furie BC, Furie B. P-selectin glycoprotein ligand 1 (PSGL-1) is a physiological ligand for E-selectin in mediating T helper 1 lymphocyte migration. J Exp Med. 2000; 192(11):1669-1675.

25. Frenette $P S$, Denis $C V$, Weiss $L$, et al. P-selectin glycoprotein ligand 1 (PSGL-1) is expressed on platelets and can mediate platelet-endothelial interactions in vivo. J Exp Med. 2000;191(8):1413-1422.

26. Wang Y, Sakuma M, Chen Z, et al. Leukocyte engagement of platelet glycoprotein Ib via integrin Mac-1 is critical for the biological response to vascular injury. Circulation. 2005;112(19):2993-3000.

27. Simon DI, Chen $\mathrm{Z}, \mathrm{Xu} \mathrm{H}$, et al. Platelet glycoprotein Iba is a counterreceptor for the leukocyte integrin Mac-1 (CD11b/CD18). J Exp Med. 2000;192(2):193-204.

28. Woodfin A, Voisin M, Imhof BA, Dejana E, Engelhardt B, Nourshargh S. Endothelial cell activation leads to neutrophil transmigration as supported by the sequential roles of ICAM-2, JAM-A and PECAM-1. Blood. 2009;113(24):6246-6257.

29. Weber A, Przytulski B, Schumacher M, et al. Flow cytometry analysis of platelet cyclooxygenase-2 expression: Induction of platelet cyclooxygenase-2 in patients undergoing coronary artery bypass grafting. Br J Haematol. 2002;117(2):424-426.

30. Dixon DA, Tolley ND, Bemis-Standoli K, et al. Expression of COX-2 in platelet-monocyte interactions occurs via combinatorial regulation involving adhesion and cytokine signaling. Clin Invest. 2006; 116(10):2727-2738.

31. Glenister KM, Payne KA, Sparrow RL. Proteomic analysis of supernatant from pooled buffy-coat platelet concentrates throughout 7-day storage. Transfusion. 2008;48(1):99-107.

32. Lindemann S, Tolley ND, Dixon DA, et al. Activated platelets mediate inflammatory signaling by regulated interleukin $1 \beta$ synthesis. J Cell Biol. 2001;154(3):485-490.

33. Beaulieu LM, Lin E, Mick E, et al. Interleukin 1 receptor 1 and interleukin $1 \beta$ regulated megakaryocyte maturation, platelet activation and transcript profile during inflammation in mice and humans. Arterioscler Thromb Vasc Biol. 2014;34(3):552-564.

34. Neumann H. Molecular mechanisms of axonal damage in inflammatory central nervous system diseases. Curr Opin Neurol. 2003;16(3): 267-273.

35. Thorsteinsdottir S, Bjerrum OW, Hasselbach HC. Myeloproliferative neoplasms in five multiple sclerosis patients. Leuk Res Rep. 2013;2(2): 61-63.

36. Christiansen CF, Christensen S, Farkas DK, Miret M, Sørensen HT, Pedersen L. Risk of arterial cardiovascular diseases in patients with multiple sclerosis: A population-based cohort study. Neuroepidemiology. 2010;35(4):267-274.

37. Jadidi E, Mohammadi M, Moradi T. High risk of cardiovascular diseases after diagnosis of multiple sclerosis. Mult Scler. 2013;19(10):1336-1340.

38. Brønnum-Hansen H, Koch-Henriksen N, Stenager E. Trends in survival and cause of death in Danish patients with multiple sclerosis. Brain. 2004;127(Pt 4):844-850.

39. Shah SM, Khan S, Rehman SU, Khan ZA, Wisal A, Wisal Z. Addressing the impact of stroke risk factors in a case control study in tertiary care hospitals: A case-control study in Tertiary Care Hospitals of Peshawar, Khyber Phukhtoonkhwa (KPK), Pakistan. BMC Res Notes. 2013;6:268.
40. Ocak G, Vossen CY, Verduijn M, et al. Risk of venous thrombosis in patients with major illnesses: Results from the MEGA study. J Thromb Haemost. 2013;11(1):116-123.

41. Arpaia G, Bavera PM, Caputo D, et al. Risk of deep venous thrombosis (DVT) in bedridden or wheelchair-bound multiple sclerosis patients: A prospective study. Thromb Res. 2010;125(4):315-317.

42. Sternberg Z, Leung C, Sternberg D, Yu J, Hojnacki D. Disease-modifying therapies modulate cardiovascular risk factors in patients with multiple sclerosis. Cardiovasc Ther. 2014;32(2):33-39.

43. Pankratz S, Bittner S, Kehrel BE, et al. The inflammatory role of platelets: Translational insights from experimental studies of autoimmune disorders. Int J Mol Sci. 2016;17(10):1723.

44. Behari M, Shrivastava M. Role of platelets in neurodegenerative diseases: A universal pathophysiology. Int J Neurosci. 2013;123(5):287-299. doi:10.3109/00207454.2012.751534

45. Nathanson M, Savitsky JP. Platelet adhesive index studies in multiple sclerosis and other neurologic disorders. Bull $N$ Y Acad Med. 1952;28(7):462-468.

46. Sheremata WA, Jy W, Horstman LL, Ahn YS, Alexander JS, Minagar A. Evidence of platelet activation in multiple sclerosis. J Neuroinflammation. 2008;5:27. doi:10.1186/1742-2094-5-27

47. Starossom SC, Veremeyko T, Yung AW, et al. Platelets play differential role during the initiation and progression of autoimmune neuroinflammation. Circ Res. 2015;117(9):779-792.

48. Morel A, Bijak M, Miller E, Rywaniak J, Miller S, Saluk J. Relationship between the increased haemostatic properties of blood platelets and oxidative stress level in multiple sclerosis patients with the secondary progressive stage. Oxid Med Cell Longev. 2015;2015:240918. doi:10.1155/2015/240918

49. Morel A, Miller E, BijakM, SalukJ.The increased level of COX-dependent arachidonic acid metabolism in blood platelets from secondary progressive multiple sclerosis patients. Mol Cell Biochem. 2016;420(1-2): 85-94. doi:10.1007/s11010-016-2770-6

50. Langer HF, Chavakis T. Platelets and neurovascular inflammation. Thromb Haemost. 2013;110(5):888-893.

51. Langer HF, Choi EY, Zhou H, et al. Platelets contribute to the pathogenesis of experimental autoimmune encephalomyelitis. Circ Res. 2012;110(9):1202-1210. doi:10.1161/CIRCRESAHA.111.256370

52. Hon GM, Hassan MS, van Rensburg SJ, Erasmus RT, Matsha T. The haematological profile of patients with multiple sclerosis. Open J Mod Neurosurg. 2012;2(3):36-44.

53. Farrokhi $M$, Beni $A A$, Etemadifar $M$, et al. Effect of fingolimod on platelet count among multiple sclerosis patients. Int J Prev Med. 2015; 6:125. doi:10.4103/2008-7802.172539

54. Obermann M, Ruck T, Pfeuffer S, Baum J, WiendI H, Meuth SG. Simultaneous early-onset immune thrombocytopenia and autoimmune thyroid disease following alemtuzumab treatment in relapsingremitting multiple sclerosis. Mult Scler. 2016;22(9):1235-1241. doi:10. $1177 / 1352458516638558$

55. May AE, Kälsch T, Massberg S, Schmidt R, Gawaz M. Engagement of glycoprotein Ilb/Illa (allb $\beta 3$ ) on platelets upregulates CD40L and triggers CD40L-dependent matrix degradation by endothelial cells. Circulation. 2002;106(16):2111-2117.

56. Gawaz M, Langer H, May AE. Platelets in inflammation and atherogenesis. J Clin Invest. 2005;115(12):3378-3384.

57. Lievens D, Zernecke A, Seijkens T, et al. Platelet CD40L mediates thrombotic and inflammatory processes in atherosclerosis. Blood. 2010;116(20):4317-4327.

58. Gerdes N, Zhu L, Ersoy M, et al. Platelets regulate CD4 ${ }^{+}$T-cell differentiation via multiple chemokines in humans. Thromb Haemost. 2011;106(2):353-362.

59. Fletcher JM, Lalor SJ, Sweeney CM, Tubridy N, Mills KH. T cells in multiple sclerosis and experimental autoimmune encephalomyelitis. Clin Exp Immunol. 2010;162(1):1-11.

60. Kempuraj D, Thangavel R, Natteru PA, et al. Neuroinflammation induces neurodegeneration. J Neurol Neurosurg Spine. 2016;1(1):1003.

61. Shahrara S, Pickens SR, Mandelin AM, et al. IL-17-mediated monocyte migration occurs partially through CCL2/MCP-1 induction. J/mmunol. 2010;184(8):4479-4487.

62. Drolet $A M$, Thivierge $M$, Turcotte $S$, et al. Platelet-activating factor induces Th17 cell differentiation. Mediators Inflamm. 2011;2011: 913802. 
63. Li N. CD4+ T cells in atherosclerosis: Regulation by platelets. Thromb Haemost. 2013;109(6):980-990.

64. Dinkla S, van Cranenbroek B, van der Heijden W, et al. Platelet microparticles inhibit IL 17 production by regulatory $T$ cells through P-selectin. Blood. 2016;127(16):1976-1986.

65. Horstman LL, Jy W, Ahn YS, et al. Role of platelets in neuroinflammation: A wide-angle perspective. J Neuroinflammation. 2010;7:10.

66. Duerschmied D, Suidan GL, Demers M, et al. Platelet serotonin promotes the recruitment of neutrophils to sites of acute inflammation in mice. Blood. 2013;121(6):1008-1015.

67. Morrell CN, Aggrey AA, Chapman LM, Modjeski KL. Emerging roles for platelets as immune and inflammatory cells. Blood. 2014;123(18) 2759-2767.
68. Centonze D, Muzio L, Rossi S, et al. Inflammation triggers synaptic alteration and degeneration in experimental autoimmune encephalomyelitis. J Neurosci. 2009;29(11):3442-3452.

69. Seizer P, May AE. Platelets and matrix metalloproteinases. Thromb Haemost. 2013;110(5):903-909.

70. Bar-Or A, Nuttall RK, Duddy M, et al. Analyses of all matrix metalloproteinase members in leukocytes emphasize monocytes as major inflammatory mediators in multiple sclerosis. Brain. 2003;126(Pt 12): 2738-2749.

71. Saluk-Juszczak J, Wachowicz B, Kaca W. Endotoxins stimulate generation of superoxide radicals and lipid peroxidation in blood platelets. Microbios. 2000;103(404):17-25.

72. Gironi M, Borgiani B, Mariani $E$, et al. Oxidative stress is differentially present in multiple sclerosis courses, early evident and unrelated to treatment. J Immunol Res. 2014;2014:961863. doi:10.1155/ 2014/961863 